\title{
Review on Agricultural Plant Disease Detection by using Image Processing
}

\author{
Priyanka L. kamble \\ Department of Electronics and Telecommunication Engineering \\ SKN College of engineering ,korti , Pandharpur, Maharashtra, India \\ Anjali C. Pise \\ Department of Electronics and Telecommunication Engineering \\ SKN college of engineering korti , Pandharpur , Maharashtra , India
}

\begin{abstract}
This paper provides review on leaf disease detection technique by using image processing. Diseases decrease the productivity of plant. Which restrict the growth of plant and quality and quantity of plant also reduces. leaf disease detection is very important research topic. Number of crops caused by fungi, bacteria etc. To overcomes this by using automatic leaf detection of plant by different image processing technique. Image processing is best way for detecting and diagnosis the diseases.
\end{abstract}

Keywords - Watermarking, Haar Wavelet, DWT, PSNR

\section{INTRODUCTION}

Research in agriculture is aimed towards increase of productivity and food quality at reduced expenditure and with increased profit. Farmers have wide range of diversity to select suitable fruit and vegetable crop. Research work develops the advance computing system to identify the diseases using infected images of various leaf spots. Such crops caused by fungi, bacteria, viruses. Disease management is a challenging task. Huge numbers of disease are seen on leafs or stems of plant Image processing is best way for detecting and diagnosis the diseases. In which initially the infected region is found then different features are extracted such as color, texture and shape. Finally classification technique is used for detecting the diseases. There are different feature extraction techniques for extracting the color, texture and edge features such as color space, color histogram, grey level co-occurrence matrix (CCM), Gabor filter, Canny and Sobel edge detector. There are also different classification techniques such as Support Vector Machine (SVM), Artificial Neural Network (ANN), Backpropagation (BP) Network, Probabilistic Neural Network (PNN), Radial Basis Function (RBF) Neural Network

This paper is organized into the following sections. Section 1 gives an introductory part includes importance of leaf disease detection, plant leaves analysis. Section 2 presents a detailed discussion on recent work carried out in this area. Section 3 includes basic methodology for leaves disease detection which represents a brief review on various image processing techniques. Finally, section 4 concludes this paper along with possible future directions.

\section{LITERATURE REVIEW}

P. Revati, et.al [1] has studied "Advance Computing Enrichment Evaluation of Cotton Leaf Spot Disease Detection Using Image Edge detection". This work express Technological Strategies using mobile captured symptoms of Cotton Leaf Spot images and categorize the diseases using neural network. The classifier is being trained to achieve intelligent farming, including early detection of disease in the groves, selective fungicide application, etc. This proposed work is based on Image Edge detection Segmentation techniques in which, the captured images are processed for enrichment first. Then R, G, B color Feature image segmentation is carried out to get target regions (disease spots). Later, image features such as boundary, shape, color and texture are extracted for the disease spots to recognize diseases and control the pest recommendation.

Zulkifli Bin Husin, et.al [2] proposed work on "Feasibility study on plant chili disease detection using image processing". This paper includes effective way used for detection of chili disease through leaf feature inspection. Leaf image is captured and processed to determine the health status of each plant. This research strongly recommends to be use for early detection of plant chili disease through leaf inspection. Leaves images captured are processed to determine the healthiness of each plant. By using leaf recognition technique, it does will identify the 
potential problems to the chili plants before its goes seriously damage for all chili plants. With this method, the use of harmful chemicals on plants can be reduced and hence ensure a healthier environment and may be even lowering the production cost of the maintenance and producing a high quality of chili.

H. Al-Hiary, et.al [3] has studied "a framework for detection and classification of plant leaf and stem diseases". They proposed faster and more accurate solution. They used K-means clustering \&back propagation feed forward neural network for detection \& classification of diseases that affect on plant leaves.

Camargoa, et.al [4] analyzed "Image pattern classification for the identification of disease causing agent in plants". It includes a machine vision system for the identification of the visual symptoms of plant diseases, from colored images. This study suggested that: texture-related features might be used as discriminators when the target images do not follow a well defined color or shape domain pattern; and that machine vision systems might lead to the successful discrimination of targets when fed with appropriate information.

Qing Yao, et.al [5] analyzed "Application of support vector machine for detecting rice diseases using shape and color texture features". This paper suggests design for Support Vector Machine (SVM) for detecting rice diseases. Rice disease spots were segmented and their shape and texture features were extracted. Because the color features are influenced largely by outside light, they selected shape and color texture features of disease spot as characteristic values of classification. The SVM method was employed to classify rice bacterial leaf blight, rice sheath blight and rice blast. The results showed that SVM could effectively detect and classify these disease spots to an accuracy of $97.2 \%$.

Geng Ying, et.al [6] “ A Study on the Method of Image Pre-Processing for Recognition of Crop Diseases” They used cucumber powdery mildew, speckle \& downy mildews as study samples \& reported comparative study of effect of simple filter and median filter. They stated that Leaves with spots must be pre-processed firstly in order to carry out the intelligent diagnosis to crop based on image processing.

Camargoa, et.al [7] has studied "Image processing -based algorithm to automatically identify plant disease visual symptoms". It includes an image-processing based method that identifies the visual symptoms of plant diseases, from an analysis of colored images. The processing algorithm developed starts by converting the RGB image of the diseased plant or leaf, into the $\mathrm{H}, \mathrm{I} 3 \mathrm{a}$ and I3b color transformations. The transformed image is then segmented by analyzing the distribution of intensities in a histogram. This technique is particularly useful when the target in the image data set is one with a large distribution of intensities.

A.Meunkaewjinda, et.al [8] proposed work on "Grape leaf disease detection from color imagery system using hybrid intelligent system". This paper includes hybrid intelligent system. Grape leaf disease is detected in from color imagery using hybrid intelligent system. They used self organizing maps \&back propagations neural networks to recognize colors of grape leaf. This information is used to segment grape leaf pixels within the image. Then the grape leaf disease segmentation is performed using modified self organizing feature maps with genetic algorithms for optimization \&support vector machines for classification. The segmented image is filtered using Gabor wavelet which allows the system to analyze leaf disease color features more efficiently. The support vector machines are then applied to classify types of grape leaf disease.

Xinhong Zhang, et.al [9] "Features Extraction of Tobacco Leaves". A system based on machine vision techniques is proposed for the automatic inspection of flue-cured tobacco leaves. Machine vision techniques are used in this system to solve problems of features extraction and analysis of tobacco leaves, which include features of color, size, shape and surface texture. The experimental results show that this system is a viable way for the features extraction of tobacco leaves, and can be used for the automatic classification of tobacco leaves.

S.S. Abu-Naser, et. Al [10] "Developing an Expert System for Plant Disease Diagnosis" the design \&development of an expert system with two different methods for diagnosing plants Diseases were presented:1) step by step descriptive method 2)graphical representation method. It is reported that the expert system with the graphical representation is more favourable it is found that the graphical representation requires few desaription from users. The praposed system saved a lot of time \& effort in identifying plant disease .

Weizheng S, et.al [11] "Grading Method Of Leaf Spot Disease Based On Image Processing" fast \& accurate novel method is developed which is based on image processing for grading of plant disease. They segmented leaf region using Otsu segmentation. The plant diseases are graded by calculating the quotient of disease spot \& leaf area.

Stephen Gang Wu, et.al [12] "A Leaf Recognition Algorithm for Plant Classification Using Probabilistic Neural Network". Paper implements a leaf recognition algorithm using easy-to-extract features and high efficient recognition algorithm. A Probabilistic Neural Network (PNN) approach for plant leaf recognition is used. The features are extracted and processed by PCA to form input to PNN. It was found that algorithm works with an accuracy of $90 \%$ on 32 kinds of plants. 


\section{BASIC PROCEDURE OF DISEASE DETECTION}

Basic flow chart for plant disease detection and classification is as follow-

Image Acquisition

Images of the infected leaves are taken from. This database has different types of plant diseases; and the images are stored in JPEG format. These images are then read in MATLAB using imread command.

Image pre processing

If noises are present in image, interested region in the image is not clear. In the image clipping, smoothing, enhancement are the three steps included in preprocessing phase. The process of image collection and lots of information may bring noise which make the quality of image dropped. To perform denoising different kinds of reduction technique are applicable.

\section{Image Segmentation}

Image segmentation is the first step and also one of the most critical tasks of image analysis. According to the region of interest, the image will be segmented into different parts. To divide the image into same meaningful region is the image segmentation.

\section{Image Feature Extraction}

The features extraction is the input data transform into set of features. The feature set will extract the relevant information so should carefully chosen. Feature extraction involves simplifying the amount of resources required to describe a large set of data accurately.

\section{Image classification}

The intent of the classification process is to categorize all pixels in a digital image into one of classes, or theme. The objective of image classification is to identify, as a unique gray level (or color), the features occurring in an image in terms of the object or type these features actually represent on the ground. Image classification is perhaps the most important part of digital image analysis. 


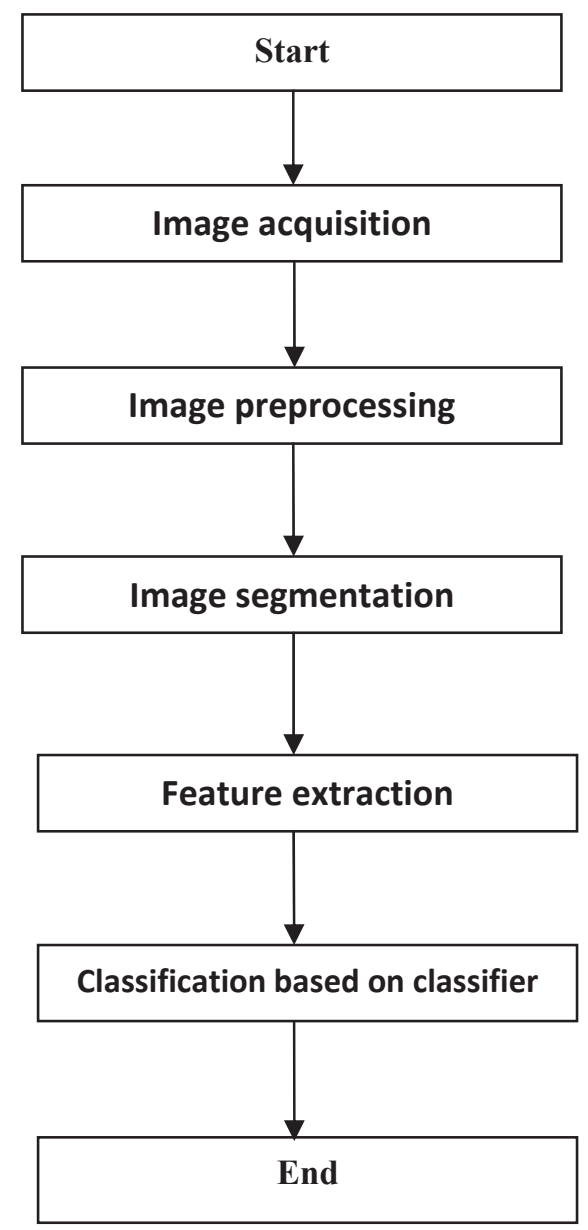

Figure 1. basic flow chart of disease detection and classification.

\section{IV.CONCLUSION}

Image processing-based approach is proposed and useful for plant diseases detection. This paper describes different techniques of image processing for several plant species that have been used for detecting plant diseases.

\section{REFERENCES}

[1] P.Revati, M. Hemlata" Advance Computing Enrichment Evaluation of Cotton Leaf Spot Disease Detection Using Image Edge detection,” IEEE 20180. July 2012

[2] Zulkifli Bin Husin, Abdul Hallis Bin Abdul Aziz, Ali Yeon Bin Md Shakaff and Rohani Binti S Mohamed Farook, "Feasibility Study on Plant Chili Disease Detection Using Image Processing Techniques," IEEE $3^{\text {rd }}$ international conference on intelligent system on modeling and simulation, pp 291- 296, 2012.

[3] H .Al-Hiary, S. Bani-Ahmad, M. Reyalat, M. Braik and Z. AlRahamneh "Framework for detection \& classification of plant leaf and stem diseases," IEEE international conference on signal and image processing, pp 113-118, 2010.

[4] Camargo A. and J. S. Smith, "Image pattern classification for the identification of disease causing agents in plants," Computer and Electronics in Agriculture, vol.66, pp 121- 125, January 2009.

[5] Qing Yao, Zexin Guan, Yingfeng Zhou, Jian Tang, Yang Hu and Baojun Yang "Application of support vector machine for detecting rice diseases using shape and color texture features," IEEE International Conference on Engineering Computation, pp 79-83,2009.

[6] Geng Ying, Li Miao, Yuan Yuan and Hu Zelin, "A Study on the Method of Image Pre-Processing for Recognition of Crop Diseases," IEEE International Conference on Advanced Computer Control, pp 202-206, 2009.

[7] Camargo, A., Smith, J., "An image processing-based algorithm to automatically identify plant disease visual symptoms," Biosystems engineering, vol.102, pp 9-21, September 2008.

[8] Meunkaewjinda, P. Kumsawat, K. Attakitmongcol and A .Srikaew, "Grape leaf disease detection from color imagery system using hybrid intelligent system," IEEE international conference, pp.513-516, 2008. 
[9] Xinhong Zhang \& Fan Zhang "Images Features Extraction of Tobacco Leaves," computer society, pp-773-776, 2008.

[10] Abu-Naser, K. A. Kashkash and M. Fayyad "Developing an Expert System for Plant Disease Diagnosis," Journal of Artificial Intelligence1(2):78-85, 2008.

[11] Weizheng S.Yachun W.,Zhanliang C.\& Hongda W “Grading Method Of Leaf Spot Disease Based On Image Processing,” Proceedings Of 2008 International Conference On Computer Science And Software Engineering, Volume 06,2008

[12] Stephen Gang Wu, Forrest Sheng Bao, Eric You Xu, Yu - Xuan Wang Yi - Fan Chang"A Leaf Recognition Algorithm for Plant Classification Using Probabilistic Neural Network,"IEEE 7th International Symposium on Signal Processing and Information Technology, 2007.

[13] Panagiotis Tzionas, Stelios E. Papadakis and Dimitris Manolakis "Plant leaves classification based on morphological features and fuzzy surface selection technique," 5th International Conference ON Technology and Automation ICTA'05, Thessaloniki, Greece, pp.365370,2005 .

[14] J. Du, D. Huang, X. Wang, and X. Gu, "Shape recognition based on radial basis probabilistic neural network and application to plant species identification" in Proceedings of International Symposium of Neural Networks, ser. LNCS 3497.Springer, 2005.

[15] Ahsan Abdullah and Muhammad Umer "Application of Remote Sensing in Pest Scouting:Evaluating Options and Exploring Possibilities", Proceedings of the 7th International Conference on Precision Agriculture and Other Precision Resources Management, Hyatt Regency, Minneapolis,MN,USA,2004. 